\title{
Critical generalized inverse participation ratio distributions
}

\author{
E. Cuevas⿵⺆ \\ Departamento de Física, Universidad de Murcia, E-30071 Murcia, Spain
}

(Dated: November 3, 2018)

\begin{abstract}
The system size dependence of the fluctuations in generalized inverse participation ratios (IPR's) $I_{\alpha}(q)$ at criticality is investigated numerically. We focus on a three-dimensional (3D) system with unitary symmetry, a 2D system with symplectic symmetry and a 1D system with orthogonal symmetry. The variances of the IPR logarithms are found to be scale-invariant at the macroscopic limit. The finite size corrections to the variances decay algebraically with nontrivial exponents, which depend on the Hamiltonian symmetry and the dimensionality. The large- $q$ dependence of the asymptotic values of the variances behaves as $q^{2}$ according to theoretical estimates. These results ensure the self-averaging of the corresponding generalized dimensions.
\end{abstract}

PACS numbers: 71.30.+h, 05.45.Df, 72.15.Rn, 73.20.Jc

The statistical properties of wave functions have been intensively studied during recent years, helping in our understanding of phenomena in different areas of physics, ranging from nuclear and $^{2}$ atomid 3 to microwave 3 and mesoscopic physics. 15 . what happens at the critical point of a metal-insulator transition (MIT), in which eigenstates presenting strong amplitude fluctuations were demonstrated to be multifractal objects.

The Anderson MIT essentially depends on the dimensionality and symmetries of the system. The field theoretical formulation 10 of the MIT shows that the critical behavior can be described within a framework of three universality classes: orthogonal $(\beta=1)$, unitary $(\beta=2)$, and symplectic $(\beta=4)$. The symmetry parameter $\beta$ is the number of independent real components that characterizes a matrix element of the corresponding Hamiltonian. The two major symmetries in field theory are time-reversal symmetry and spin-rotation symmetry. The system belongs to the orthogonal class if it has both symmetries, to the unitary class if time-reversal symmetry is broken, and to the symplectic class if the system has time-reversal symmetry but spin-rotation is broken. The relevant terms in the hamiltonian are a coupling to an applied magnetic field, which breaks time-reversal symmetry, and the spin-orbit interaction, which breaks spinrotation symmetry.

Castellani and Peliti11 considered the $\phi=2+\epsilon(\epsilon \ll 1)$ expansion of Wegner's nonlinear $\sigma$ mode 12 for the generalized inverse participation ratios (IPR's) of eigenstates,

$$
I_{\alpha}(q)=\int_{\Omega} d^{d} r\left|\psi_{\alpha}(\mathbf{r})\right|^{2 q} \propto L^{-d_{q}(q-1)},
$$

and concluded that the wave functions $\psi_{\alpha}(\mathbf{r})$ at the transition point of a MIT show multifractal behavior characterized by an infinite set of generalized fractal dimensions $d_{q}$. The index $\alpha$ labels the different eigenfunctions and $\Omega$ denotes a $d$-dimensional region with linear dimension $L$. Equation (值) is valid for individual states and for their ensemble average since the spectrum of multifractal dimensions has universal features for states in the vicinity of the MITL.

As regards the IPR fluctuations, we shall first review the existing analytical results. When these fluctuations were studied for two-dimensional systems in the framework of the supersymmetry method $\$ 14$ it was found that the distribution function of $I_{\alpha}(q)$, normalized to its typical value, is scale-invariant at criticality.14 Although this case does not present a true Anderson transition, the above result motivated the conjecture that in general the normalized distribution function of $I_{\alpha}(q)$ is universal, i.e., size independent for $L \rightarrow \infty$. Accordingly, it is assumed that the distribution function of $\ln I_{\alpha}(q)$ is a universal curve that, while horizontally shifted by changes in $L$, keeps the same form. Within the same approach, Mirlin and Evers 15.16 study the fractal properties of the power-law random banded matrix (PRBM) ensemble at criticality. This model is characterized by a parameter, $b$ [see Eq. (7) below]. In the two limiting cases, $b \gg 1$ and $b \ll 1$, for which analytical treatment is feasible, they found the distribution function of the IPR to be scaleindependent at the macroscopic limit. Particularly, they analyzed the behavior of the variance $\sigma_{z_{q}}^{2}$ of the distribution function of $I_{\alpha}(q)$, normalized to its typical value, in the limit $b \ll 1$. For values of $q$ larger than some critical value, $q_{\mathrm{c}} \approx 2.41$, where the IPR distribution is dominated by its slowly decaying power law tail, the same authors obtained for $L \rightarrow \infty$

$$
\sigma_{z_{q}}^{2}(\infty)=\left(q / q_{\mathrm{c}}\right)^{2}
$$

What we are trying to point out is that a general analytical description of the statistical properties of the generalized IPR at the critical point of realistic MIT's (conventional Anderson transition, quantum Hall transition, transition in $d=2$ for electrons with strong spin-orbit coupling, etc.) is still lacking. This is why we addressed the problem using numerical calculations. To our knowledge, no such calculations have been previously reported.

Our aim was to find the system size dependence of the generalized IPR distributions in order to check whether they converge at large $L$ to the conjectured scale invariant distributions or not. A second motivation for this work was to clarify whether the corresponding general- 
ized dimensions are well-defined in the macroscopic limit or presents scale-invariant distributions.

Using the exact eigenstates from numerical diagonalizations, we obtained the size dependence of the distribution function $\mathcal{P}_{\beta}\left(z_{q}=\ln I_{\alpha}(q)\right)$ at the critical point of a three-dimensional (3D) system with unitary symmetry, a two-dimensional (2D) system with symplectic symmetry and a one-dimensional (1D) system with orthogonal symmetry. All these systems possess a mobility edge and are modeled by the corresponding tight-binding Hamiltonian. We found that as $L$ increases $\mathcal{P}_{\beta}$ becomes wider and the height of its peak smaller with a tendency towards saturation. Therefore, their variance increases as $L$ increases and will eventually saturate to a constant finite value for $L \rightarrow \infty$. In order to extrapolate to macroscopic systems, we propose a finite size correction to the variance $\sigma_{z_{q}}^{2}$ of $z_{q}$ according to

$$
\sigma_{z_{q}}^{2}(L)=\sigma_{z_{q}}^{2}(\infty)-a_{q} L^{-y_{q}}
$$

with $\sigma_{z_{q}}^{2}(\infty), a_{q}$ and $y_{q}>0$ being three adjustable parameters. Practically for all triads $\{q, \beta, d\}$ we found that the exponent $y_{q}$ was always close to the generalized dimension $d_{q}$ divided by $2 \beta d$. Thus, for the exponent $y_{q}$ we propose the following equation,

$$
y_{q}=\frac{d_{q}}{2 \beta d},
$$

and keep only two free parameters. This relation constitutes the main result of the present publication. As we will see below, our numerical data strongly support this conjecture. Equation (伍) is a generalization of the exponent $\gamma=d_{2} / 2 d$ found by our group 17 for $I_{\alpha}(2)$ in disordered systems with orthogonal symmetry.

Some technical details follow: the critical eigenfunctions (and eigenvalues) of the Hamiltonian matrices, Eqs. (5), (6), and (7), are obtained by numerical diagonalization. In $3 \mathrm{D}$ and $2 \mathrm{P}$ systems we use techniques for large sparse matrices 18 while for the $1 \mathrm{D}$ case standard diagonalization subroutines are used, since we must deal with full matrices. We consider a small energy window $(-\eta, \eta)$ around the center of the spectral band, where $\eta=1(\eta=0.4)$ for 3D and 2D (1D) systems. Reducing the width of the above windows does not alter the results. For an accurate extrapolation to the thermodynamic limit, the system size changes by one order of magnitude for $3 \mathrm{D}$ and $2 \mathrm{D}$ systems and by two orders in the case of 1D. The number of random realizations is such that the number of critical states included for each $L$ is roughly $1.5 \times 10^{5}$, while, in order to reduce edge effects, we impose periodic boundary conditions in all the cases considered. We have checked that the normalized nearest level variances 19 are indeed scale-invariant at each critical point studied. Below we present our numerical results and compare them to the conjecture (位).

$3 D$ unitary symmetry. First we focus on the breaking of time-reversal symmetry by a constant applied magnetic field. We consider the standard Anderson model

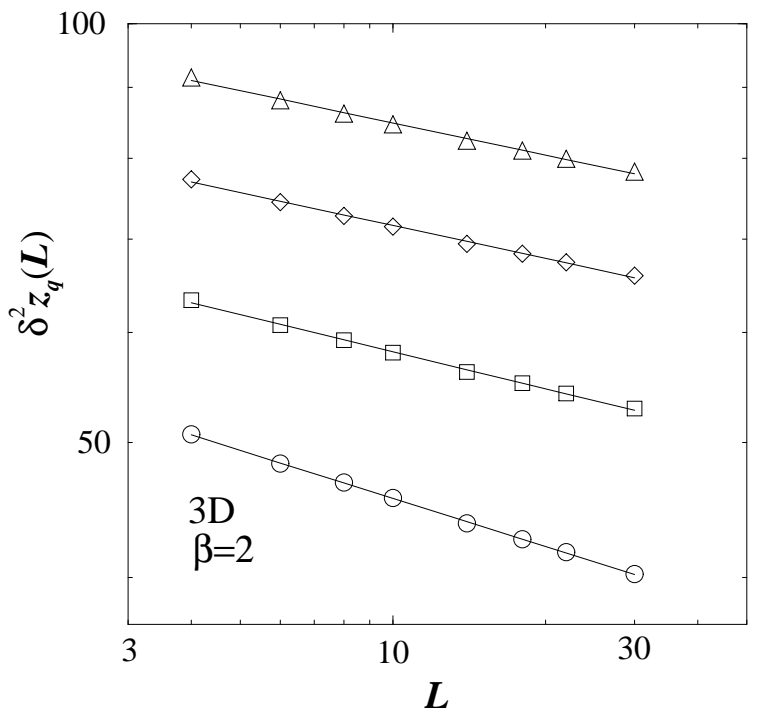

FIG. 1: Finite size correction $\delta^{2} z_{q}(L)$ as a function of system size $L$ for the 3D disordered system with unitary symmetry and several $q$ values: $q=2$ (circles), $q=4$ (squares), $q=6$ (diamonds), and $q=8$ (triangles). The straight lines are linear fits to Eq. (3).

with diagonal disorder and Peierls phase factors in the hopping matrix elements

$$
\mathcal{H}=\sum_{n} \epsilon_{n} c_{n}^{\dagger} c_{n}+\sum_{n, m} V_{n m} c_{n}^{\dagger} c_{m}
$$

where $c_{n}^{\dagger}\left(c_{n}\right)$ are the creation (annihilation) operators of an electron at a site $n$ of a simple-cubic lattice, and the summation in the second term is over nearest neighbors. The diagonal energies are randomly distributed with constant probability in the interval $-W / 2<\epsilon_{n}<W / 2$, where $W$ parametrizes the strength of random potential. Energies are measured in units of the modulus of the hopping matrix elements, $V_{n m}$, and lengths in units of the lattice constant. The hopping matrix elements, $V_{n m}=e^{\mp 2 \pi i \alpha z}$ for neighbors in the $y$ direction and $V_{n m}=1$ in any other direction, describe a system with a homogeneous magnetic field, $B$, in the $x$ direction, while the Peierls phase $\alpha=e B / h c$ is the number of flux quanta $\Phi_{0}=h c / e$ threading a lattice cell. Here, the Landau gauge with the vector potential $\mathbf{A}=(0,-B z, 0)$ is chosen. The system size varies between 4 and 30 , and for the critical disorder we take the value $W_{\mathrm{c}}=18.35$ reported in Ref. 20 for $\alpha=0.25$.

In Fig. 1 we represent on a log-log scale the finite size correction $\delta^{2} z_{q}(L) \equiv \sigma_{z_{q}}^{2}(\infty)-\sigma_{z_{q}}^{2}(L)$ as a function of $L$ for the 3D disordered system with unitary symmetry and different values of $q$ : 2 (circles), 4 (squares), 6 (diamonds), and 8 (triangles). The good agreement with Eqs. (3) and (4) is evident. The fitted values of the free parameters are $a_{2}=2.01, a_{4}=19.71, a_{6}=52.88$, $a_{8}=100.7$, while the corresponding $\sigma_{z_{q}}^{2}(\infty)$ are summa- 
rized in Fig. 4 (squares). The slopes of the straight lines have not been fitted and correspond to Eq. (4), with $\beta=2, d=3, d_{2}=1.37, d_{4}=1.07, d_{6}=0.98$, and $d_{8}=0.94$. These generalized dimensions were estimated from the slope, $d_{q}(1-q)$, of $\left\langle z_{q}\right\rangle$ versus $\ln L$. In order to produce a diagram of reasonable size the curves for $q=2$, 4 , and 6 have been shifted vertically. The small difference in the slopes for $q \geq 4$ correspond to the slight differences in the values of $d_{q}$. The value $\sigma_{z_{2}}(\infty)=1.40$ found is in good agreement with the conjecture $\sigma_{z_{2}}(\infty) \sim 1$.

$2 D$ symplectic symmetry. In order to calculate the critical eigenstates (two-component spinors) Ando's model Hamiltonian 21 was used,

$$
\mathcal{H}=\sum_{n, \sigma} \epsilon_{n} c_{n, \sigma}^{\dagger} c_{n, \sigma}+\sum_{n, \sigma, m, \sigma^{\prime}} V_{n, \sigma ; m, \sigma^{\prime}} c_{n, \sigma}^{\dagger} c_{m, \sigma^{\prime}}
$$

where $c_{n, \sigma}^{\dagger}\left(c_{n, \sigma}\right)$ are the creation (annihilation) operators of an electron at a site $n$ of a square lattice with the spin component $\sigma$ and $m$ denotes the sites adjacent to site $n$. The on-site energy $\epsilon_{n}$ is randomly distributed around zero according to a box distribution with a width $W$, which specifies the degree of disorder. The index $\sigma$ takes on values of +1 and -1 , and denotes spin-up or spin-down. The transfer matrices $V_{n, \sigma ; m, \sigma^{\prime}}=V_{x}$ or $V_{y}$ depend on the direction of the nearest neighbor site

$$
V_{x}=\left(\begin{array}{cc}
V_{1} & V_{2} \\
-V_{2} & V_{1}
\end{array}\right), \quad V_{y}=\left(\begin{array}{cc}
V_{1} & -i V_{2} \\
-i V_{2} & V_{1}
\end{array}\right)
$$

and the strength of the spin-orbit coupling is given by the parameter $S=V_{2} / V$, with $V=\left(V_{1}^{2}+V_{2}^{2}\right)^{1 / 2}$ taken to be the unit of energy. We chose $S=1 / 2$ and, for the critical disorder, $W_{\mathrm{c}}=5.98$, which was previously found in Ref. 22 . The system size ranges from $L=10$ to $L=120$.

Figure 2 shows the same size corrections as Fig. 1 for the 2D disordered system with symplectic symmetry. In this case the fitted values of the free parameters are $a_{2}=0.17, a_{4}=5.10, a_{6}=15.77$, and $a_{8}=31.27$ and the values of $\sigma_{z_{q}}^{2}(\infty)$ are shown in Fig. 4 (circles). As in the $3 \mathrm{D}$ case, the slopes of the straight lines correspond to Eq. (4), with $\beta=4, d=2, d_{2}=1.60, d_{4}=1.34, d_{6}=1.23$, and $d_{8}=1.18$. These dimensions were also estimated in a similar way. Again, the value found, $\sigma_{z_{2}}(\infty)=0.46$, is in agreement with the conjecture $\sigma_{z_{2}}(\infty) \sim 1$.

$1 D$ orthogonal symmetry. In this case the PRBM model15. 16 is used. The Hamiltonian, which describes a disordered 1D sample with random long-range hopping, is represented by real symmetric matrices, whose entries are randomly drawn from a normal distribution with zero mean $\left\langle\mathcal{H}_{n m}\right\rangle=0$, and a variance depending on the distance between lattice sites

$$
\left\langle\left|\mathcal{H}_{n m}\right|^{2}\right\rangle=\frac{1}{1+(|n-m| / b)^{2}} .
$$

The model describes a whole family of critical theories parametrized by $b$, which determines the critical dimensionless conductance, in the same way as the dimensionality labels the different Anderson transitions. In the

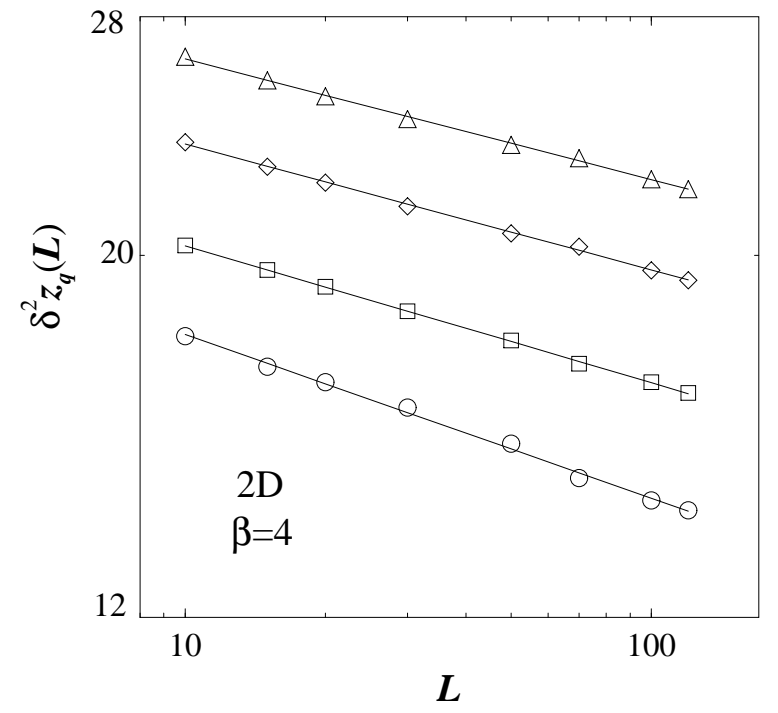

FIG. 2: As for Fig. 1, for the 2D disordered system with symplectic symmetry.

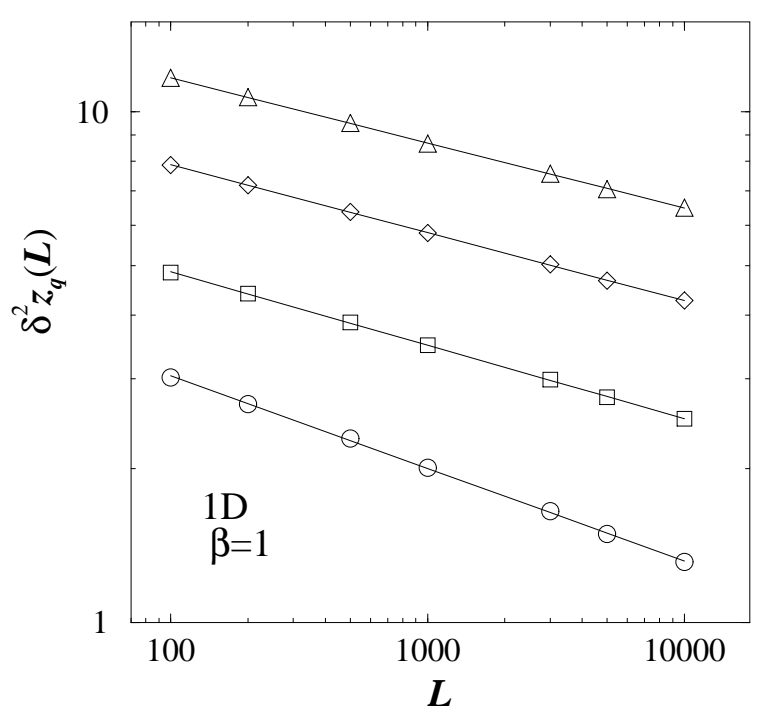

FIG. 3: As for Fig. 1, for the 1D disordered system with orthogonal symmetry.

intermediate regime $b \sim 1$, where there is no analytical solution, we obtained two different diverging exponents for the sprrelation and localization lengths at the critical region. ${ }^{3} 3$ The system size ranges between $L=100$ and $L=10000$, and for the calculations we choose $b=0.2$.

Figure 3 shows the same size corrections as Fig. 1 for the 1D disordered system with orthogonal symmetry. The fitted values of the free parameters are $a_{2}=0.47$, $a_{4}=4.28, a_{6}=11.16, a_{8}=20.95$, and $\sigma_{z_{q}}^{2}(\infty)$ are shown in Fig. 4 (diamonds). As in the two previous cases, the slopes of the straight lines correspond to Eq. (四), with 


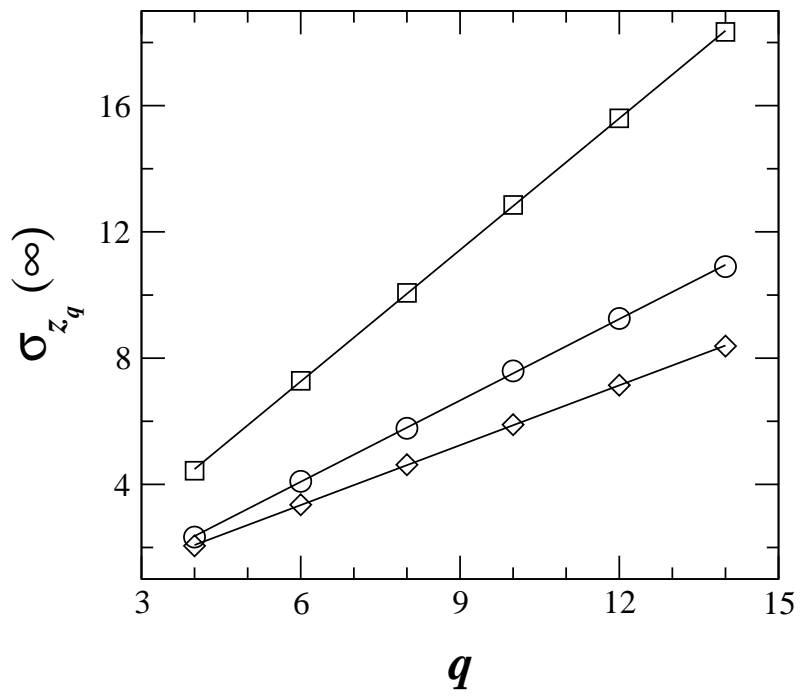

FIG. 4: Asymptotic values of the standard deviation $\sigma_{z_{q}}(\infty)$ as a function of $q$ for the $1 \mathrm{D}$ system with orthogonal symmetry (diamonds), 2D symplectic (circles), and 3D unitary (squares).

$\beta=1, d=1, d_{2}=0.36, d_{4}=0.29, d_{6}=0.27$, and $d_{8}=0.26$. The last four dimensions were estimated in a way similar to those for Fig. 1. As in the $3 \mathrm{D}$ and $2 \mathrm{D}$ cases the value $\sigma_{z_{2}}(\infty)=0$ f 7 found is in agreement with the conjecture $\sigma_{z_{2}}(\infty) \sim 1 . \mathrm{G}$

We checked that Eqs. (3) and (4) adequately describe the IPR fluctuations in the standard 3D Anderson transition (orthogonal symmetry) and in the critical point of the 1D system with long-range hopping, Eq. (7), when unitary and symplectic symmetries are included. We are confident that further analytical development in IPR statistics at criticality will confirm our conjectured result.

The statistical properties of wave functions are intimately related to the universal conductance fluctuations. As was shown in Ref. 6 , the relative variance of $I_{\alpha}(2)$ is of the order of $1 / g^{2}, g$ being the dimensionless conductance (in units of $e^{2} / h$ ). The extrapolation of this relation to the transition point, in which $g \sim 1$, is the most direct connection between our results and experiments. In this way, our results could be checked in experiments with random systems (semiconductor heterostructures, metal oxide semiconductor MOS inversion layers, vapordeposited films, doped and amorphous semiconductor, etc.) using samples with varying size.

Finally, we focus on the $q$ dependence of the asymptotic values $\sigma_{z_{q}}^{2}(\infty)$ for each universality class. In Fig. 4 we represent the standard deviation $\sigma_{z_{q}}(\infty)$ versus $q$ for the three transitions studied: 1D orthogonal (diamonds), 2D symplectic (circles), and 3D unitary (squares). The slopes of the fitted straight lines are $0.63,0.86$, and 1.39 , respectively. It is interesting to note that the nonlinear $\sigma$ model estimates, Eq. (2), of the 1D PRBM model is valid for all three transitions. These results further support our conjecture, described by Eq. (4). The different values of the slopes directly reflect the universality class and the dimensionality of the system. From the above slope for the $1 \mathrm{D}$ system we obtain $q_{\mathrm{c}}=1.59$, which is less than the value of 2.41 predicted theoretically. The origin of the deviation is that the latter value of $q_{\mathrm{c}}$ was obtained for the distribution function of $I_{\alpha}(q)$ normalized to its typical value, while the former $q_{\mathrm{c}}$ corresponds to the distribution function of $\ln I_{\alpha}(q)$. Anyway, we have estimated the value of $q_{\mathrm{c}}$ from the extrapolated long tails of the normalized $I_{\alpha}(q)$ distributions. The obtained value $q_{\mathrm{c}}=2.21$ is in close agreement with the predicted one for these distributions.

If we now assume that the fluctuations of the generalized dimensions $d_{q}$ are due to fluctuations of the corresponding generalized IPR, 24 from Eq. (11) we obtain $\sigma_{d_{q}}^{2}(L)=\sigma_{z_{q}}^{2}(L) /(q-1)^{2} \ln ^{2} L$ for the variance of $d_{q}$. From this relation, and taking into account Eqs. (2), (3), and (任), we see that $\sigma_{z_{q}}(L)$ vanishes at a rate $1 / \ln L$ when $L \rightarrow \infty$. Therefore, we conclude that all generalized dimensions are self-averaged quantities, i.e., they tend to well-defined single values at the macroscopic limit.

In summary, we have performed a detailed numerical analysis of the statistical properties of the IPR distributions at the critical point of three standard MIT's, finding the finite asymptotic values of the variances of the distributions in each case. The corresponding finite size corrections decay with system size as a power law with exponents that depend on the system dimensionality and on the Hamiltonian symmetry. For large values of $q$, the asymptotic values $\sigma_{z_{q}}^{2}(\infty) \propto q^{2}$ for all three transitions is in agreement with the analytical estimates of the 1D PRBM model. Therefore, all generalized dimensions are well-defined at the macroscopic limit.

We would like to thank the Spanish DGESIC, Project No. 1FD97-1358 and No. BFM2000-1059, for financial support.
* Electronic address: ecr@um.es; URL: http://bohr.fcu. un.es/miembros/ecr/

1 V. Zelevinsky, B. A. Brown, N. Frazier, and M. Horoi, Phys. Rep. 276, 85 (1996).

2 A. F. Brunello, T. Uzer, and D. Farrelly, Phys. Rev. Lett. 76, 2874 (1996).
3 P. Pradhan and S. Sridhar, Phys. Rev. Lett. 85, 2360 (2000).

4 B. L. Altshuler, V. E. Kravtsov, and I. V. Lerner, in Mesoscopic Phenomena in Solids, edited by B. L. Altshuler, P. A. Lee, and R. A. Webb (North Holland, Amsterdam, 1991). 
${ }^{5}$ B. A. Muzykantskii and D. E. Khmelnitskii, Phys. Rev. B 51, 5480 (1995).

6 Y.V. Fyodorov and A.D. Mirlin, Phys. Rev. B 51, 13403 (1995).

7 B.L. Altshuler and L.S. Levitov, Phys. Rep. 288, 487 (1997).

8 A.D. Mirlin, Phys. Rep. 326, 259 (2000).

9 C. Chamon, C. Mudry, and X.G. Wen, Phys. Rev. Lett. 77, 4194 (1996); H.E. Castillo et al., Phys. Rev. B 56, 10668 (1997).

10 K.B. Efetov, Adv. Phys. 32, 53 (1983).

11 C. Castellani and L. Peliti, J. Phys. A 19, L429 (1986).

12 F. Wegner, Z. Phys. B 36, 209 (1980).

13 M. Janssen, Int. J. Mod. Phys. B 8, 943 (1994).

14 V.N. Prigodin and B.L. Altshuler, Phys. Rev. Lett. 80, 1944 (1998).

15 F. Evers and A.D. Mirlin, Phys. Rev. Lett. 84, 3690 (2000).

16 A.D. Mirlin and F. Evers, Phys. Rev. B 62, 7920 (2000).

17 E. Cuevas, M. Ortuño, V. Gasparian, and A. Pérez-
Garrido, Phys. Rev. Lett. 88, 016401 (2002).

18 J.K. Cullum and R.A. Willoughby, Lanczos Algorithms for Large Symmetric Eigenvalue Computations (Birkhauser, Basel, 1985).

19 E. Cuevas, Phys. Rev. Lett. 83, 140 (1999); E. Cuevas, E. Louis, and J.A. Vergés, ibid. 77, 1970 (1996).

20 T. Dröse, M. Batsch, I. Kh. Zharekeshev, and B. Kramer, Phys. Rev. B 57, 37 (1998).

21 T. Ando, Phys. Rev. B 40, 5325 (1989).

${ }^{22}$ L. Schweitzer and I. Kh. Zharekeshev J. Phys.: Condens. Matter 9, L441 (1997).

23 E. Cuevas, V. Gasparian, and M. Ortuño, Phys. Rev. Lett. 87, 056601 (2001).

${ }^{24}$ V. E. Kravtsov, in Correlated Fermions and Transport in Mesoscopic Systems, edited by T. Martin, G. Montambaux, and J. Tran Tanh Van (Editions Frontieres, Gif-surYvette, 1996). 